\title{
Efficacy of breast MRI for surgical decision in patients with breast cancer: ductal carcinoma in situ versus invasive ductal carcinoma
}

Jeeyeon Lee ${ }^{1}$, Jin Hyang Jung ${ }^{1}$, Wan Wook Kim', Chan Sub Park' ${ }^{1}$ Ryu Kyung Lee', Hye Jung Kim², Won Hwa Kim ${ }^{2}$ and Ho Yong Park ${ }^{1,3^{*}}$

\begin{abstract}
Background: Preoperative breast magnetic resonance imaging (MRI) provides more information than mammography and ultrasonography for determining the surgical plan for patients with breast cancer. This study aimed to determine whether breast MRI is more useful for patients with ductal carcinoma in situ (DCIS) lesions than for those with invasive ductal carcinoma (IDC).

Methods: A total of 1113 patients with breast cancer underwent mammography, ultrasonography, and additional breast MRI before surgery. The patients were divided into 2 groups: DCIS $(n=199)$ and IDC $(n=914)$, and their clinicopathological characteristics and oncological outcomes were compared. Breast surgery was classified as follows: conventional breast-conserving surgery (Group 1), partial mastectomy with volume displacement (Group 2), partial mastectomy with volume replacement (Group 3), and total mastectomy with or without reconstruction (Group 4). The initial surgical plan (based on routine mammography and ultrasonography) and final surgical plan (after additional breast MRI) were compared between the 2 groups. The change in surgical plan was defined as group shifting between the initial and final surgical plans.
\end{abstract}

Results: Changes (both increasing and decreasing) in surgical plans were more common in the DCIS group than in the IDC group $(P<0.001)$. These changes may be attributed to the increased extent of suspicious lesions on breast MRI, detection of additional daughter nodules, multifocality or multicentricity, and suspicious findings on mammography or ultrasonography but benign findings on breast MRI. Furthermore, the positive margin incidence in frozen biopsy was not different $(P=0.138)$.

Conclusions: Preoperative breast MRI may provide more information for determining the surgical plan for patients with DCIS than for those with IDC.

Keywords: Breast, Ductal carcinoma, Magnetic resonance imaging, Surgical plan

\footnotetext{
* Correspondence: knuh_bts@naver.com

'Department of Surgery, School of Medicine, Kyungpook National University, Daegu, Republic of Korea

${ }^{3}$ Department of Surgery, Joint Institute for Regenerative Medicine, School of

Medicine, Kyungpook National University, Hoguk-ro 807, Buk-gu, Daegu

41404, Republic of Korea

Full list of author information is available at the end of the article
}

(C) The Author(s). 2020 Open Access This article is licensed under a Creative Commons Attribution 4.0 International License, which permits use, sharing, adaptation, distribution and reproduction in any medium or format, as long as you give appropriate credit to the original author(s) and the source, provide a link to the Creative Commons licence, and indicate if changes were made. The images or other third party material in this article are included in the article's Creative Commons licence, unless indicated otherwise in a credit line to the material. If material is not included in the article's Creative Commons licence and your intended use is not permitted by statutory regulation or exceeds the permitted use, you will need to obtain permission directly from the copyright holder. To view a copy of this licence, visit http://creativecommons.org/licenses/by/4.0/ The Creative Commons Public Domain Dedication waiver (http://creativecommons.org/publicdomain/zero/1.0/) applies to the data made available in this article, unless otherwise stated in a credit line to the data. 


\section{Background}

Preoperative breast magnetic resonance imaging (MRI) is an optional modality for the evaluation of breast cancer. However, compared with mammography or ultrasonography, it can provide additional information for diagnosing ductal carcinoma in situ (DCIS) [1-3]. In addition, the involvement of the nipple or nipple-areolar complex in breast cancer can be easily detected with additional breast MRI [4, 5]. The usefulness of breast MRI has been demonstrated among Asian women who have dense breasts or are BRCA mutation carriers with a higher risk of contralateral breast cancer [6, 7].

The surgical plan for breast cancer is usually determined according to the excision volume, tumor location, glandular density, and ratio of tumor to whole breast volume [8, 9]. The tumor extent, ductal pattern, existence of daughter nodules, and multifocality or multicentricity can be detected in additional breast MRI. However, because the characteristics of images differ depending on the tumor type, the imaging modality to be performed should be carefully determined. Although DCIS has excellent prognosis, the excision volume is usually larger than that of a single nodule of invasive ductal carcinoma (IDC) due to the ductal pattern $[2,10]$. In triple-negative breast cancer, the breast lesion may appear round, which can be misinterpreted as a benign lesion [11-13]. In those cases, additional preoperative breast MRI can provide more important information.

In this study, we evaluated the usefulness of preoperative breast MRI in determining the surgical plan for patients with breast cancer [14]. Although this result was not described in detail in our previous study, we found that changes in surgical plans were more common among patients with carcinoma in situ lesions than among those with invasive carcinoma (13.0\% vs. 9.9\%). Hence, this study aimed to determine whether breast MRI is more useful in determining the surgical plan for patients with DCIS than for those with IDC.

\section{Methods}

Between 2006 and 2014, the medical records and oncological status of 1327 patients with operable, primary breast cancer who underwent cancer surgery at Kyungpook National University Hospital (Daegu, Republic of Korea) were reviewed. Among them, 1113 patients with ductal carcinoma underwent mammography, breast ultrasonography, and MRI before surgery. Breast MRI was performed with the patient prone using a $1.5 \mathrm{~T}$ system (Signa Excite; GE Medical Systems, Milwaukee, WI) with a dedicated 4-channel breast coil. Each patient was given $0.1 \mathrm{~mL} / \mathrm{kg}$ gadolinium-diethylenetriamine pentaacetate (Magnevist; Schering, Berlin, Germany) as the contrast agent, which was injected at a rate of $1 \mathrm{~mL} / \mathrm{s}$. Axial T1-weighted images (repetition time [TR]/echo time [TE], 416/10; matrix, $320 \times 224$; slice thickness, 3.4 $\mathrm{mm}$ ) and sagittal fat-suppressed T2-weighted images (TR/TE, 3000/94; matrix, $320 \times 224$; slice thickness, 2.6 $\mathrm{mm})$ were acquired. Dynamic contrast-enhanced magnetic resonance examination included 1 precontrast and 5 postcontrast images with bilateral sagittal acquisition by 3-dimensional gradient-echo fat-suppressed T1weighted imaging (TR/TE, 6.2/2.9; matrix, $288 \times 416$; flip angle, $10^{\circ}$; slice thickness, $2.6 \mathrm{~mm}$ ). The patients were divided into 2 groups (DCIS and IDC) based on needle biopsy results (Fig. 1). The study protocol was approved by the Institutional Review Board Committee of Kyungpook National University Hospital (2016-10-008).

The treatment for breast cancer was determined by the combined opinion of a multidisciplinary team including breast and plastic surgeons, oncologists, radiologists, pathologists, and radiation oncologists. Based on the resection volume of the breast, the types of surgery were classified as follows: breast-conserving surgery (Group 1), partial mastectomy with volume displacement (Group 2), partial mastectomy with volume replacement (Group 3), and total mastectomy with or without breast reconstruction (Group 4).

The initial surgical plan was determined based on mammography and ultrasonography findings, and the final surgical plan was determined based on additional breast MRI findings and second-look ultrasonography results when an additional suspicious lesion was detected on the breast MRI. When an additional suspicious lesion (detected on breast MRI and second-look ultrasonography) was present in different quadrants of the breast, biopsy was performed. Both surgical plans were compared to determine whether the interventions involved were the same. The change in surgical plan was defined as group shifting between the initial and final surgical plans.

The inclusion criteria for this study were as follows: 1) Biopsy revealed malignant disease with ductal origin; and 2) additional breast MRI was conducted before surgery and deciphered by highly experienced radiologists. Patients whose biopsy result was reported as other malignant breast diseases excluding ductal carcinoma, who developed other primary malignancies, and who underwent neoadjuvant chemotherapy or excisional biopsy before breast MRI were excluded. Regardless of DCIS or IDC, when the final diagnosis after surgery was different from the initial diagnosis, those patients were also excluded. In addition, if an extensive intraductal component was found based on the result of needle biopsy for IDC, those patients were excluded to reduce bias. For the evaluation of the surgical margin status, breast tissues were obtained in at least four different directions from the cavity and used to prepare frozen sections and permanent sections. Surgical margin positivity was 


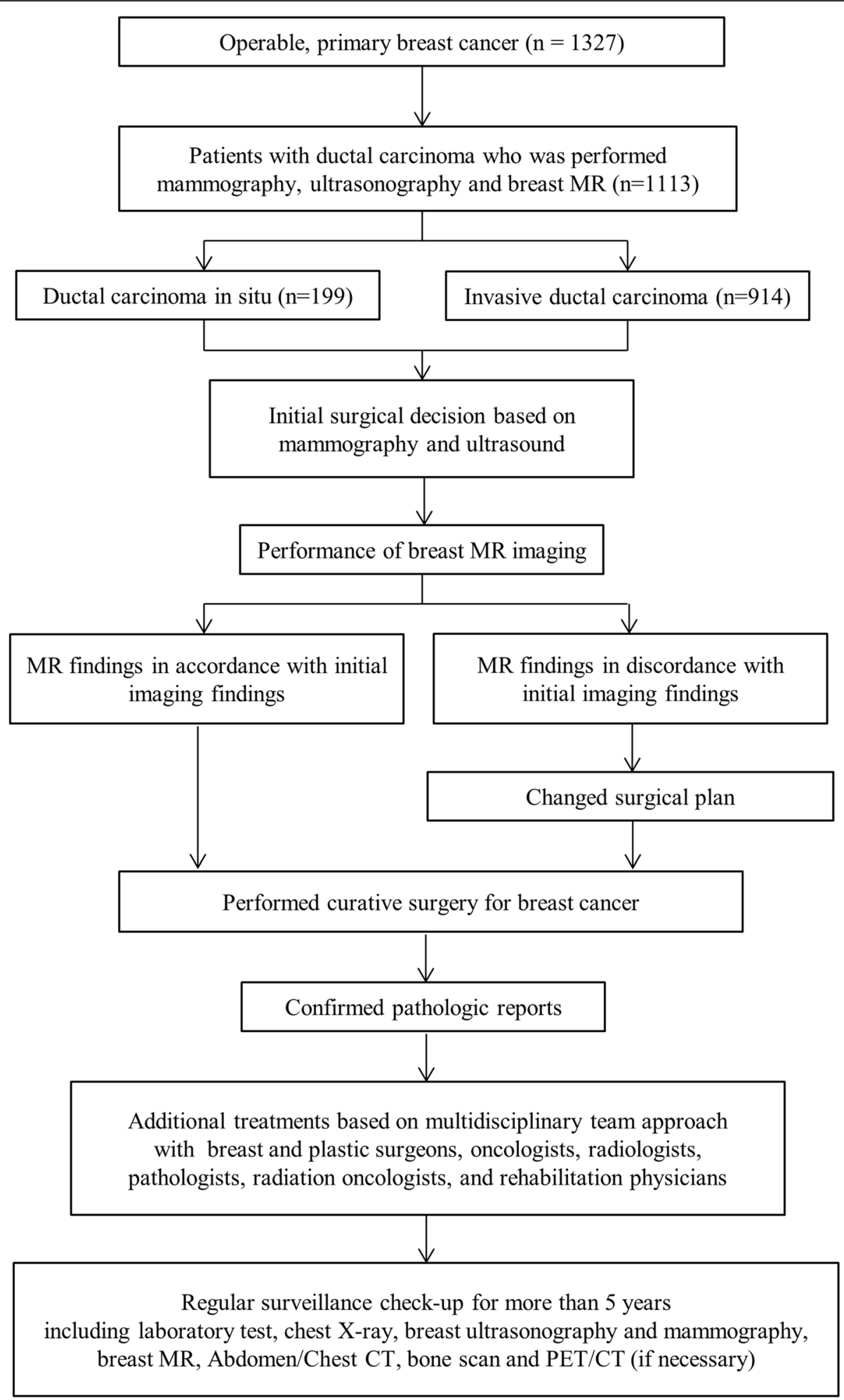

Fig. 1 A flowchart demonstrating the process of changing surgical plans based on the results of mammography, ultrasonography, and additional breast magnetic resonance imaging, as well as the management of breast cancer using a multidisciplinary team approach 
defined as the presence of atypical cells, carcinoma in situ, or invasive cancer cells on the cut surface. After the negative results of the surgical margin were confirmed, reconstructive surgery was performed.

Patients who requested to change their surgical plan regardless of imaging findings or those whose surgical plan was changed because of the previous excision status (not because of breast MRI findings) were included in the patient group with changes in surgical plans but were excluded from the group when changes in surgical plans were related to MRI results. Additional treatments including adjuvant chemotherapy, radiotherapy, hormone therapy, or targeted treatment were administrated based on the patients' tumor characteristics.

The follow-up period was extended to at least 4 years after the initial treatment. The oncological outcomes were evaluated to determine the rate of locoregional recurrence, distant metastasis, and mortality. Regular surveillance was performed every 6 months during the first 2 years and annually after 3 years, and included laboratory blood test with tumor markers, chest $\mathrm{x}$-ray, mammography, breast ultrasonography, thoracic and abdominal computed tomography, bone scan, and positron emission tomography/computed tomography (if necessary).

The clinicopathological factors were obtained from medical records, and statistical analysis was performed using SPSS (version 23; SPSS Inc., Chicago, IL, USA). Quantitative and categorical variables were compared using Student's $t$ test and the $x^{2}$ test, respectively. A $P$ value of less than 0.05 was considered significant.

\section{Results}

There were no significant differences in the mean age, mean body mass index, and clinical and pathological tumor size between the DCIS and IDC groups. The incidence of bilateral breast cancer was higher in the DCIS group (DCIS, $7.0 \%$ vs. IDC, $3.3 \% ; P=0.014$ ), and triplenegative breast cancer was more frequent in the IDC group (DCIS, $5.5 \%$ vs. IDC, 9.3\%; $P=0.002$ ). No significant difference was observed in the rate of locoregional recurrence between the 2 groups $(P=0.506)$. The prevalence of distant metastasis was significantly higher in the IDC group $(P<0.001)$ (Table 1$)$.

The incidence of increased surgical scale was greater in the DCIS group (DCIS, $14.0 \%$ vs. IDC, $8.9 \% ; P=0.002)$. In addition, the incidence of decreased surgical scale was greater in the DCIS group (DCIS, $2.0 \%$ vs. IDC, $0.8 \% ; P=0.035$ ) (Fig. 2). Therefore, significant differences were observed in the proportion of patients with changes in surgical plans between the DCIS and IDC groups $(P<0.001)$.

The increase in the surgical scale may be attributed to the following observations: increased extent of suspicious lesions on breast MRI compared with mammography and ultrasonography (DCIS, 9.0\% vs. IDC, 7.1\%), additional daughter nodules on breast MRI (DCIS, 3.5\% vs. IDC, $1.4 \%$ ), and multifocality or multicentricity on breast MRI (DCIS, $1.5 \%$ vs. IDC, $0.3 \%$ ). However, the

Table 1 Clinicopathological characteristics of patients with breast cancer who were diagnosed with ductal carcinoma in situ and invasive ductal carcinoma

\begin{tabular}{|c|c|c|c|}
\hline Characteristics & $\begin{array}{l}\text { Ductal carcinoma in situ } \\
(n=199)\end{array}$ & $\begin{array}{l}\text { Invasive ductal carcinoma } \\
(n=914)\end{array}$ & $P$ value \\
\hline Mean age (years, \pm SD) & $50.1 \pm 9.4$ & $49.3 \pm 9.9$ & 0.649 \\
\hline Mean body mass index $\left(\mathrm{kg} / \mathrm{m}^{2}, \pm \mathrm{SD}\right)$ & $23.2 \pm 3.0$ & $23.4 \pm 3.2$ & 0.745 \\
\hline History of bilateral breast cancer (n, \%) & $14(7.0)$ & $30(3.3)$ & 0.014 \\
\hline Clinical tumor size on ultrasound $(\mathrm{cm}, \pm \mathrm{SD})$ & $2.01 \pm 1.7$ & $2.0 \pm 1.2$ & 0.213 \\
\hline Pathological tumor size $(\mathrm{cm}, \pm \mathrm{SD})$ & $2.2 \pm 1.9$ & $1.7 \pm 1.1$ & 0.109 \\
\hline Estrogen receptor, positive (n, \%) & $135(67.8)$ & $639(69.9)$ & 0.379 \\
\hline Progesterone receptor, positive $(n, \%)$ & $120(60.3)$ & $568(62.1)$ & 0.136 \\
\hline c-erbB2 protein, positive (n, \%) & $73(36.7)$ & $169(18.5)$ & 0.226 \\
\hline Triple-negative breast cancer (n, \%) & $11(5.5)$ & $85(9.3)$ & 0.002 \\
\hline Adjuvant chemotherapy (n, \%) & 0 & $460(50.3)$ & $<0.001$ \\
\hline Adjuvant radiotherapy (n, \%) & $67(33.7)$ & $636(69.6)$ & $<0.001$ \\
\hline Adjuvant hormonal therapy (n, \%) & $125(62.8)$ & $687(75.2)$ & 0.061 \\
\hline Follow-up period (mo, \pm SD) & $90.1 \pm 25.3$ & $88.6 \pm 19.1$ & 0.241 \\
\hline Locoregional recurrence (n, \%) & $3(1.5)$ & $17(1.9)$ & 0.506 \\
\hline Distant metastasis (n, \%) & $1(0.5)$ & $24(2.6)$ & $<0.001$ \\
\hline Death (n, \%) & $1(0.5)$ & $9(1.0)$ & 0.192 \\
\hline
\end{tabular}




\begin{tabular}{|c|c|c|c|c|c|c|c|c|}
\hline \multirow{3}{*}{$\begin{array}{c}\text { Initial } \\
\text { decision }\end{array}$} & \multicolumn{8}{|c|}{ Final decision } \\
\hline & \multicolumn{4}{|c|}{ Ductal carcinoma in situ $(\mathrm{n}=199)$} & \multicolumn{4}{|c|}{ Invasive ductal carcinoma $(\mathrm{n}=914)$} \\
\hline & Group 1 & Group 2 & Group 3 & Group 4 & Group 1 & Group 2 & Group 3 & Group 4 \\
\hline Group 1 & $86(43.2)$ & $4(2.0)$ & 0 & $12(6.0)$ & $480(52.5)$ & $19(2.1)$ & $7(0.8)$ & $9(1.0)$ \\
\hline Group 2 & $1(0.5)$ & $16(8.0)$ & $1(0.5)$ & $11(5.5)$ & 0 & $126(13.8)$ & $8(0.9)$ & $21(2.3)$ \\
\hline Group 3 & $3(1.5)$ & 0 & $8(4.0)$ & 0 & $1(0.1)$ & $4(0.4)$ & $68(7.4)$ & $17(1.9)$ \\
\hline Group 4 & 0 & 0 & 0 & $57(28.6)$ & 0 & $1(0.1)$ & $1(0.1)$ & $152(16.6)$ \\
\hline \multirow{2}{*}{\multicolumn{5}{|c|}{$\begin{array}{l}\text { Group 1: Breast conserving surgery } \\
\text { Group 2: Partial mastectomy with volume displacement technique } \\
\text { Group 3: Partial mastectomy with volume replacement technique } \\
\text { Group 4: Total mastectomy with or without reconstruction }\end{array}$}} & & & \multirow{2}{*}{\multicolumn{2}{|c|}{$\begin{array}{l}\text { Increased surgical scales } \\
\text { Decreased surgical scales }\end{array}$}} \\
\hline & & & & & & & & \\
\hline
\end{tabular}

Fig. 2 Group shifting of breast surgery based on breast magnetic resonance imaging findings of patients with ductal carcinoma in situ and invasive ductal carcinoma. The gray box represents the group with a higher surgical scale, and the dotted box represents the group with a lower surgical scale

suspicious lesions detected on mammography or ultrasonography appeared benign on breast MRI. Hence, the surgical scale was decreased (DCIS, $2.0 \%$ vs. IDC, $0.8 \%$ ). Six patients in the DCIS group and 4 patients in the IDC group requested to change the surgical plan regardless of imaging findings. However, in pathological evaluation, the incidence of positive margin in the initial frozen biopsy in Groups 1, 2, and 3 and nipple margin in Group 4 was not different between the 2 groups $(P=0.470$ and 0.101) (Table 2).

The pathological findings, which were matched to preoperative breast MRI findings, are shown in Table 3.
Based on the final pathological reports, the number of cases showing a larger tumor size than that observed on mammography or ultrasonography was $14(43.8 \%)$ in the DCIS group and $49(55.7 \%)$ in the IDC group $(P=0.784)$. The pathological evaluation revealed true malignancy in DCIS $(n=9,28.1 \%)$ and IDC $(n=32,36.3 \%)$. However, among IDC cases showing true malignancy, the background of the DCIS component was observed in 27 cases (30.7\%). In addition, benign pathological findings were found in DCIS $(n=5,15.6 \%)$ and IDC $(n=17,19.3 \%)$.

In the IDC group, 6 (6.8\%) cases involved multiple lymphovascular invasion and $9(10.2 \%)$ demonstrated an

Table 2 Changes in the surgical scale and reasons for the changes in the surgical plans of patients with ductal carcinoma in situ and invasive ductal carcinoma

\begin{tabular}{|c|c|c|c|}
\hline & $\begin{array}{l}\text { Ductal carcinoma in situ } \\
(n=199)\end{array}$ & $\begin{array}{l}\text { Invasive ductal carcinoma } \\
(n=914)\end{array}$ & $P$ value \\
\hline Increased surgical scale (n, \%) & $28(14.0)$ & $81(8.9)$ & 0.002 \\
\hline Decreased surgical scale (n, \%) & $4(2.0)$ & $7(0.8)$ & 0.035 \\
\hline Total number of cases with surgical plan changes $(n, \%)$ & $38(19.1)$ & $92(10.1)$ & $<0.001$ \\
\hline Changes in surgical plans based on MRI findings (n, \%) & $32(16.1)$ & $88(9.6)$ & $<0.001$ \\
\hline \multicolumn{4}{|l|}{ Reasons (n, \%) } \\
\hline Increased extent of suspicious lesions on breast MRI & $18(9.0)$ & $65(7.1)$ & - \\
\hline Additional lesions on breast MRI & $7(3.5)$ & $13(1.4)$ & - \\
\hline Multifocality or multicentricity on breast MRI & $3(1.5)$ & $3(0.3)$ & - \\
\hline $\begin{array}{l}\text { Suspicious lesions on mammography or ultrasonography but benign lesions on } \\
\text { breast MRI }\end{array}$ & $4(2.0)$ & $7(0.8)$ & - \\
\hline Changes in surgical plans due to patient's desire & $6(3.0)$ & $4(0.4)$ & - \\
\hline Positive margin status in the initial frozen biopsy (Groups 1-3) & $13(6.5)$ & $59(6.5)$ & 0.470 \\
\hline Positive results in nipple frozen method (Group 4) & $7(3.5)$ & $15(1.6)$ & 0.1301 \\
\hline
\end{tabular}


Table 3 Additional pathological results excluding the main lesion for cases with changes in the surgical plans based on MRI findings

\begin{tabular}{|c|c|c|c|}
\hline Additional pathological results $(\mathrm{n}, \%)$ & $\begin{array}{l}\text { Ductal carcinoma in situ } \\
(n=32)\end{array}$ & $\begin{array}{l}\text { Invasive ductal carcinoma } \\
(n=88)\end{array}$ & $P$ value \\
\hline $\begin{array}{l}\text { Larger tumor size observed on breast MRI vs. breast mammography or } \\
\text { ultrasonography }\end{array}$ & $14(43.8)$ & $49(55.7)$ & 0.784 \\
\hline True malignancy & $9(28.1)$ & $32(36.3)$ & \\
\hline Background of ductal carcinoma in situ $(>5 \mathrm{~cm})$ & - & $27(30.7)$ & \\
\hline Benign pathologic findings & $5(15.6)$ & $17(19.3)$ & \\
\hline Multiple lymphovascular invasion & - & $6(6.8)$ & \\
\hline Extensive intraductal component & - & $9(10.2)$ & \\
\hline Multifocality & $12(37.5)$ & $20(22.7)$ & 0.139 \\
\hline Separate nodules with invasive and non-invasive focus & - & $3(3.4)$ & \\
\hline Microcalcification, both in tumor and benign ducts & $6(18.8)$ & $18(20.5)$ & 0.950 \\
\hline Microcalcification, only in benign ducts & - & $7(8.0)$ & \\
\hline Sclerosing adenosis or fibroadenoma & $4(12.5)$ & $2(2.3)$ & 0.061 \\
\hline
\end{tabular}

Factors could be duplicated

MRI Magnetic resonance imaging

extensive intraductal component. In the IDC group, the background of DCIS was found in 27 (30.7\%) cases, and separate nodules with IDC and a noninvasive focus were found in $3(3.4 \%)$ cases. The number of cases with microcalcifications, which were detected in both the tumor and benign ducts, was $6(18.8 \%)$ in the DCIS group and $18(20.5 \%)$ in the IDC group $(P=0.950)$. Several benign lesions were also detected in pathological findings.

Benign lesions including microcalcifications in benign ducts, sclerosing adenosis, and fibroadenoma were observed in $4(12.5 \%)$ cases of DCIS and $9(10.3 \%)$ cases of IDC $(P=0.061)$. In addition, these findings were matched to the suspicious lesions on preoperative breast MRI.

\section{Discussion}

In comparison with mammography or ultrasonography, breast MRI is considered a superior imaging modality for assessing the extent of DCIS and detecting contralateral breast cancer during screening, which could improve the accuracy of therapeutic planning [15-17]. In particular, patients with pure DCIS may benefit more from breast MRI than from mammography, and this can be similarly useful when a DCIS component is combined with an IDC lesion [18].

However, based on hormonal or histopathological changes, the uptake of contrast in breast MRI could vary [19]. We have previously reported that breast MRI in triple-negative breast cancer is helpful for determining the surgical plan [14]. As a further study, we compared the changes in surgical plan between the IDC and DCIS groups, which were significantly more prevalent in the DCIS group.
Mammography is usually considered as a useful screening imaging modality for early breast cancer because clustered microcalcification is the most common mammographic finding among patients with DCIS [20, 21]. However, in non-mass-forming DCIS, which is common, the extent of the tumor is difficult to assess with only mammography or ultrasonography [22, 23]. Additional breast MRI may contribute to the accurate assessment of the extent of the DCIS focus or detection of occult breast cancer lesions in patients. As invasive breast cancer forms a mass or lump with typical malignant ultrasonographic features, including a hypoechoic mass with an irregular or spiculated margin, a lobulated shape with an indistinct margin, or a taller-thanwide shape [24], the extent of invasive breast cancer could be well identified by ultrasonography. However, when the DCIS component or extensive intraductal component is combined with invasive breast cancer, the extent of the breast cancer would be difficult to determine with mammography or ultrasonography.

The surgical plan for breast cancer can be established in more detail when more information is obtained from various images [25]. In current study, we found that the range of surgery, regardless increasing or decreasing, showed more changes in DCIS than IDC cases. Based on the pathophysiology of DCIS, the tumor starts from the ductal epithelium and tends to grow according to the ductal pattern. Therefore, DCIS usually appears as a clumped or linear enhancement or a non-mass enhancement on breast MRI without definite mass formation [20]. A recent study found that breast MRI was considerably more helpful in determining the surgical plan for the DCIS group. Additional breast MRI provided more information for surgical decision not only in cases with a higher surgical scale, but also in cases with a lower 
surgical scale. This finding suggests that compared with mammography or ultrasonography, breast MRI can better differentiate between benign and suspicious lesions in DCIS.

Based on several reports in the literature, the positive margin rate is significantly lower in cases in which preoperative MRI was conducted for breast cancer [1, 25]. However, in case the surgical margin is revealed as positive during the surgery, most surgeons would perform additional excision until a negative margin is confirmed. Therefore, in those cases, the oncological results would not be different in groups with MRI verses those without.

Occasionally, benign lesions appear similar to suspicious lesions on breast MRI. In a recent study, microcalcifications in benign ducts or sclerosing lesions including adenoma and fibroadenoma showed a similar pattern to that of malignant lesions on preoperative breast MRI. As a result, excision surgery was performed in these cases. However, there is no specific method to differentiate between a true malignant lesion and a benign lesion that could appear suspicious on breast MRI. Further studies are needed to improve the accuracy of differentiating these lesions before determining the surgical plan.

Although preoperative breast MRI can provide more information for determining the surgical range with high sensitivity, some investigators do not recommend breast MRI as a diagnostic imaging modality for breast cancer. Moreover, the detection rate can vary widely $(40-100 \%)$ [26-31]. Therefore, breast MRI requires an experienced radiologist who can accurately interpret the images and determine the degree of suspicion of background breast parenchyma and contralateral breast parenchyma.

There are several limitations in current study. The number of DCIS cases was much lower than that of IDC cases. In addition, the surgical plans were decided not only reflect the information from images, but also based on the opinion of the patient through the discussion.

However, for breast cancer diagnosed as DCIS on needle biopsy and showing indistinct margins on mammography or ultrasonography, additional breast MRI is helpful for determining the surgical plan. In addition, it is beneficial in cases of invasive breast cancer with an intraductal component.

\section{Conclusion}

Additional breast MRI could be more useful in determining the surgical plan for patients with DCIS than for those with IDC. In addition, this method would be useful for patients diagnosed with IDC with background DCIS components. However, our findings do not suggest that breast MRI should be performed for all patients with breast cancer. In further studies, investigators need to determine which patients can benefit most from breast MRI.

\section{Abbreviations}

DCIS: Ductal carcinoma in situ; IDC: Invasive ductal carcinoma; MRI: Magnetic resonance imaging

\section{Acknowledgements}

This study was presented at the International Society of Surgery, 48th World Congress of Surgery by Ho Yong Park.

\section{Authors' contributions}

Guarantor of the integrity of the study: JL; Study concept: JL, HYP, HJK; Study design: JL. JHJ, WWK; Definition of intellectual content: JL, RKL, CSP; Literature research: RKL, CSP, WHK, YSC, JDY; Clinical studies: JYP, J-YP, WHK, HJK, JWL, JSL; Data acquisition: WWK, YSC, SJL; Data analysis: JL; Manuscript preparation: JL, HYP; Manuscript editing: JHJ, YSC; and Manuscript review: HJK, WHK. All authors have read and approved the manuscript.

\section{Funding}

This work was supported by a National Research Foundation of Korea (NRF) grant funded by the Korean government (nos. 2014R1A5A2009242,

2019R1F1A1063853) and by a grant from the National R\&D Program for Cancer Control, Ministry of Health and Welfare, Republic of Korea (no.

1420040). This research was also supported by a grant from the Korea Health Technology R\&D Project through the Korea Health Industry Development Institute (KHIDI), funded by the Ministry of Health \& Welfare, Republic of Korea (no. HI17C1142). This work was supported by an NRF grant funded by the Korean government (MSIT) (no. NRF-2019R1A2C1006264). This work was supported by an NRF grant funded by the Korean government (no. 2017M3A9G8083382)

The funder had no role in the design of the study and collection, analysis, and interpretation of data and in writing the manuscript.

\section{Availability of data and materials}

The datasets generated and/or analyzed during the current study are not publicly available. However, they are available from the corresponding author on reasonable request.

Ethics approval and consent to participate Informed consent was obtained from all patients, and the protocol used in this study was approved by the Institutional Review Board Committee of Kyungpook National University Chilgok Hospital, Daegu, Republic of Korea (no. 2016-10-008). In addition, the specific inclusion and exclusion criteria were defined in the approved Institutional Review Board protocol. Informed consent was obtained from all patients by written document.

Consent for publication

Not applicable.

Competing interests

The authors declare that they have no competing interests.

\section{Author details}

'Department of Surgery, School of Medicine, Kyungpook National University, Daegu, Republic of Korea. ${ }^{2}$ Department of Radiology, School of Medicine, Kyungpook National University, Daegu, Republic of Korea. ${ }^{3}$ Department of Surgery, Joint Institute for Regenerative Medicine, School of Medicine, Kyungpook National University, Hoguk-ro 807, Buk-gu, Daegu 41404, Republic of Korea.

Received: 22 November 2019 Accepted: 21 September 2020

Published online: 29 September 2020

\section{References}

1. Kuhl CK, Strobel K, Bieling H, et al. Impact of Preoperative breast MR imaging and MR-guided surgery on diagnosis and surgical outcome of women with invasive breast Cancer with and without DCIS component. Radiology. 2017;284:645-55.

2. Petrillo A, Fusco R, Petrillo M, et al. Added value of breast MRI for Preoperative diagnosis of ductal carcinoma in situ: diagnostic performance on 362 patients. Clin Breast Cancer. 2017;17:e127-34. 
3. Hlawatsch A, Teifke A, Schmidt M, Thelen M. Preoperative assessment of breast cancer: sonography versus MR imaging. AJR Am J Roentgenol. 2002; 179:1493-501

4. Ponzone R, Maggiorotto F, Carabalona S, et al. MRI and intraoperative pathology to predict nipple-areola complex (NAC) involvement in patients undergoing NAC-sparing mastectomy. Eur J Cancer. 2015;51:1882-9.

5. Machida Y, Shimauchi A, Igarashi T, et al. Preoperative breast MRI: reproducibility and significance of findings relevant to nipple-areolar complex involvement. Breast Cancer. 2018;25:456-63.

6. Bick U. Intensified surveillance for early detection of breast cancer in highrisk patients. Breast Care (Basel). 2015;10:13-20.

7. Lee MV, Katabathina VS, Bowerson ML, et al. BRCA-associated cancers: role of imaging in screening, diagnosis, and management. Radiographics. 2017 37:1005-23.

8. Asgeirsson KS, Rasheed T, McCulley SJ, Macmillan RD. Oncological and cosmetic outcomes of oncoplastic breast conserving surgery. Eur J Surg Oncol. 2005;31:817-23.

9. Clough KB, Ihrai T, Oden S, et al. Oncoplastic surgery for breast cancer based on tumour location and a quadrant-per-quadrant atlas. $\mathrm{Br}$ J Surg. 2012;99:1389-95.

10. Marcotte-Bloch C, Balu-Maestro C, Chamorey E, et al. MRI for the size assessment of pure ductal carcinoma in situ (DCIS): a prospective study of 33 patients. Eur J Radiol. 2011;77:462-7.

11. Dogan BE, Turnbull LW. Imaging of triple-negative breast cancer. Ann Oncol. 2012;23(Suppl 6):vi23-9.

12. Dogan BE, Gonzalez-Angulo AM, Gilcrease $M$, et al. Multimodality imaging of triple receptor-negative tumors with mammography, ultrasound, and MRI. AJR Am J Roentgenol. 2010;194:1160-6.

13. Kojima Y, Tsunoda H. Mammography and ultrasound features of triplenegative breast cancer. Breast Cancer. 2011:18:146-51.

14. Lee J, Jung JH, Kim WW, et al. The role of preoperative breast magnetic resonance (MR) imaging for surgical decision in patients with triple-negative breast cancer. J Surg Oncol. 2016;113:12-6.

15. Lehman CD. Magnetic resonance imaging in the evaluation of ductal carcinoma in situ. J Natl Cancer Inst Monogr. 2010;2010:150-1.

16. Berg WA, Gutierrez L, NessAiver MS, et al. Diagnostic accuracy of mammography, clinical examination, US, and MR imaging in preoperative assessment of breast cancer. Radiology. 2004;233:830-49.

17. Lehman CD, Gatsonis C, Kuhl CK, et al. MRI evaluation of the contralateral breast in women with recently diagnosed breast cancer. N Engl J Med. 2007;356:1295-303.

18. Kuhl CK, Schrading S, Bieling HB, et al. MRI for diagnosis of pure ductal carcinoma in situ: a prospective observational study. Lancet. 2007;370:48592.

19. Mayrhofer RM, Ng HP, Putti TC, Kuchel PW. Magnetic resonance in the detection of breast cancers of different histological types. Magn Reson Insights. 2013;6:33-49.

20. Shiraishi A, Kurosaki Y, Maehara T, et al. Extension of ductal carcinoma in situ: histopathological association with MR imaging and mammography. Magn Reson Med Sci. 2003;2:159-63.

21. Evans AJ, Wilson AR, Burrell HC, et al. Mammographic features of ductal carcinoma in situ (DCIS) present on previous mammography. Clin Radiol. 1999:54:644-6.

22. Cho KR, Seo BK, Kim CH, et al. Non-calcified ductal carcinoma in situ: ultrasound and mammographic findings correlated with histological findings. Yonsei Med J. 2008;49:103-10.

23. Fiaschetti $V$, Pistolese CA, Perretta $T$, et al. 3-5 BI-RADs microcalcifications: correlation between MRI and histological findings. ISRN Oncol. 2011;2011: 643890.

24. Kim YR, Kim HS, Kim HW. Are irregular Hypoechoic breast masses on ultrasound always malignancies?: a pictorial essay. Korean J Radiol. 2015;16: 1266-75.

25. Pinker K, Preoperative MRI. Improves surgical planning and outcomes for ductal carcinoma in situ. Radiology. 2020;295:304-6.

26. Gilles $\mathrm{R}$, Meunier M, Lucidarme $\mathrm{O}$, et al. Clustered breast microcalcifications: evaluation by dynamic contrast-enhanced subtraction MRI. J Comput Assist Tomogr. 1996;20:9-14.

27. Frykberg ER, Bland Kl. Overview of the biology and management of ducta carcinoma in situ of the breast. Cancer. 1994;74:350-61.

28. Orel SG, Schnall MD, LiVolsi VA, Troupin RH. Suspicious breast lesions: MR imaging with radiologic-pathologic correlation. Radiology. 1994;190:485-93.
29. Pediconi F, Marzocca F, Cavallo Marincola B, Napoli A. MRI-guided treatment in the breast. J Magn Reson Imaging. 2018;48:1479-88.

30. Boetes C, Barentsz JO, Mus RD, et al. MR characterization of suspicious breast lesions with a gadolinium-enhanced TurboFLASH subtraction technique. Radiology. 1994;193:777-81.

31. Turnbull L, Brown S, Harvey I, et al. Comparative effectiveness of MRI in breast cancer (COMICE) trial: a randomised controlled trial. Lancet. 2010;375: $563-71$

\section{Publisher's Note}

Springer Nature remains neutral with regard to jurisdictional claims in published maps and institutional affiliations.
Ready to submit your research? Choose BMC and benefit from:

- fast, convenient online submission

- thorough peer review by experienced researchers in your field

- rapid publication on acceptance

- support for research data, including large and complex data types

- gold Open Access which fosters wider collaboration and increased citations

- maximum visibility for your research: over $100 \mathrm{M}$ website views per year

At BMC, research is always in progress.

Learn more biomedcentral.com/submissions 